\title{
Robust Hybrid Precoding for Interference Exploitation in Massive MIMO Systems
}

\author{
Ganapati Hegde, Student Member, IEEE, Christos Masouros, Senior Member, IEEE, Marius
}

Pesavento, Member, IEEE,

\begin{abstract}
In this paper, we consider a multiuser massive MIMO system with hybrid analog-digital precoding architecture. The phase shifters in the hybrid precoding architecture are assumed to be imperfect, where the true values of both phase and magnitude of the phase shifters are different from their nominal values. For a given analog precoding matrix, we develop an iterative algorithm to compute robust digital precoders based on the interference exploitation approach to eliminate any potential symbol errors due to the phase shifter impairments. Numerical experiments demonstrate the performance of the proposed algorithm and show its advantage over a conventional robust precoding technique.
\end{abstract}

Index Terms-Constructive interference, Semi-infinite program, Robust precoding, Hybrid precoding, Massive MIMO

\section{INTRODUCTION}

Massive multiple-input multiple-output is a promising technology to significantly enhance the spectral efficiency of the $5 \mathrm{G}$ and future cellular networks [1-4]. In this system, the base stations (BSs) are equipped with hundreds of antenna elements. This large number of antenna elements can be utilized for extensive spatial multiplexing, e.g., in the downlink lineof-sight scenario, the BSs form narrow transmit beams using precoding techniques to maximize the useful signal powers at the intended users or minimize the interference powers at the unintended users. The conventional precoding [5-7], in which each antenna is equipped with a dedicated radio-frequency (RF) chain, can tremendously increase the hardware cost and operational power in a massive MIMO system [8, 9]. To overcome this challenge, the hybrid analog-digital precoding architecture is proposed for massive MIMO systems [1013]. In this new architecture, the BSs are quipped with a significantly smaller number of RF chains than the number of antenna elements, and each RF chain is connected to multiple antenna elements through phase shifter (PS) components, as shown in Fig. 1. A PS can alter the phase of the incoming signal; however, it can not alter the magnitude of the signal.

A major drawback of the hybrid precoding is its reduced energy efficiency due to the limited degrees of freedom resulting from a smaller number of RF chains and constant gains of the PSs [14-16]. To address this challenge, the constructive interference $(\mathrm{CI})$-based precoding technique [1720] has been extended to hybrid precoding architecture in

Ganapati Hegde and Marius Pesavento are with the Communication Systems Group, Technische Universität Darmstadt, Darmstadt 64283, Germany. (e-mail: hegde@nt.tu-darmstadt.de; pesavento@nt.tu-darmstadt.de). Christos Masouros is with the Department of Electronic \& Electrical Engineering, University College London, London WC1E7JE, U.K. (e-mail: c.masouros@ucl.ac.uk)

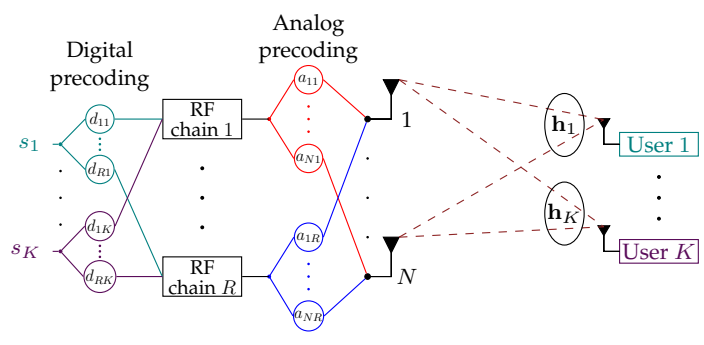

Fig. 1: Hybrid analog-digital precoding architecture.

[21-23]. In CI-based precoding, the knowledge of the current transmit symbols and channel state information are exploited to design the transmit signals such that the received signals at the users lie in the appropriate regions, known as CI-regions $[17,22,24]$. Even though the CI-based hybrid precoding improves the energy-efficiency significantly, this technique is highly sensitive to errors in the PS components of the hybrid precoding architecture, which can lead to unacceptably high symbol error rates (SERs). In [22], a CI-based hybrid precoding algorithm is proposed that computes the precoders that are robust against phase errors in the PSs. The resulting worst-case robust precoders eliminate the symbol errors resulting from the phase errors. However, the magnitude errors associated with the PSs-which are often encountered in practical PSs [25-27]—are completely neglected in [22]. In this paper, we propose a CI-based hybrid precoding algorithm that incorporates robustness against both phase and magnitude errors of the PSs into the precoders. First, we mathematically model the potential phase and magnitude errors in the PSs, and accordingly formulate the robust CI-based hybrid precoding problem. Subsequently, we extend the algorithm proposed in [22] to compute the worst-case robust precoders that are robust against the magnitude and phase errors of the PSs. In the numerical results, first, we analyze the SER increment at the users due to PS errors in the case of non-robust hybrid precoding. Furthermore, we study the performance of the proposed algorithm in terms of SER and transmit power. We also compare the performance of the proposed algorithm with that of a conventional robust precoding scheme.

\section{SySTEM MODEL}

Consider a co-channel multiuser MIMO downlink system consisting of a BS equipped with $N$ transmit antennas and $R$ RF chains, where $R \leq N$. Let $\mathcal{K} \triangleq\{1, \ldots, K\}$ denote a set of $K$ single antenna users served by the BS. The transmit symbol vector at the $\mathrm{BS}$ is given by $\mathbf{s} \triangleq\left[s_{1}, \ldots, s_{K}\right]^{\top}$, where the element $s_{k}$ indicates the symbol intended for the $k$ th user. The 


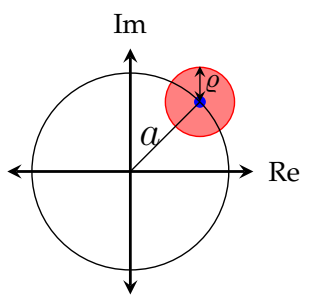

Fig. 2: The bounded error of the PS around its nominal value.

symbols are assumed to be drawn from an $M$-ary phase-shift keying ( $M$-PSK) constellation. ${ }^{1}$ Without loss of generality (w.l.o.g.) each transmit symbol is assumed to be of constant unit modulus. A digital precoder $\mathbf{d}_{k} \in \mathbb{C}^{R}$ is applied to the transmit symbol $s_{k}$ and the resulting signals are fed to $R \mathrm{RF}$ chains. Each RF chain is connected to all transmit antennas through analog PSs. Let $p_{n r}$ denote the PS that connects the $n$th antenna element to the $r$ th RF chain. The nominal (designed) value of PS $p_{n r}$ is denoted by $a_{n r}$. The PSs have constant gains, and w.l.o.g. the gains of all PSs are assumed to be identical, which is denoted by $a$. Let $\mathbf{a}_{r} \triangleq\left[a_{1 r}, \ldots, a_{N r}\right]^{\top}$ indicate the corresponding analog precoder applied to the output of the $r$ th RF chain for $r \in \mathcal{R} \triangleq\{1, \ldots, R\}$, and $\mathbf{A} \triangleq\left[\mathbf{a}_{1}, \ldots, \mathbf{a}_{R}\right]$ be the analog precoding matrix. The PSs are assumed to be imperfect, i.e., their actual values can differ from their nominal values [29-31]. Let $\tilde{a}_{n r} \triangleq a_{n r}+e_{n r}$ denote the actual value of the PS $p_{n r}$, where $e_{n r}$ denotes the error (mismatch). We assume that the magnitude of the error is bounded within a known bound $\varrho$, i.e., $\left|e_{n r}\right| \leq \varrho$, as shown in Fig. 2. The matrix of error elements $e_{n r}, \forall n \in \mathcal{N}, \forall r \in \mathcal{R}$ is represented by $\mathbf{E}$. Let $\mathcal{E}$ denote the infinite set of all possible error matrices that are associated with the nominal analog precoding matrix $\mathbf{A}$, which is given by

$$
\mathcal{E} \triangleq\left\{\mathbf{E}\left|\mathbf{E} \in \mathbb{C}^{N \times R},\right| e_{n r} \mid \leq \varrho, \forall n \in \mathcal{N}, \forall r \in \mathcal{R}\right\} .
$$

Let $\tilde{\mathbf{h}}_{k} \in \mathbb{C}^{N}$ be the frequency-flat channel vector between the BS and the $k$ th user, which is assumed to be known at the BS [2, 32]. Let $n_{k} \sim \mathcal{C N}\left(0, \sigma_{k}^{2}\right)$ represent the i.i.d. additive white Gaussian noise at the $k$ th user. The received signal $y_{k}$ at the $k$ th user can be expressed as

$$
y_{k}=\tilde{\mathbf{h}}_{k}^{\top}(\mathbf{A}+\mathbf{E})\left(\sum_{\ell=1}^{K} \mathbf{d}_{\ell} s_{\ell}\right)+n_{k}, \quad \forall k \in \mathcal{K},
$$

where the error matrix $\mathbf{E} \in \mathcal{E}$.

\section{Problem Formulation}

In this paper, we propose a technique to compute CIbased hybrid precoders that are robust against both phase and magnitude errors at the PSs, given by set $\mathcal{E}$ in (1). Our objective is to design hybrid precoders with the minimum transmit power at the BS, such that the received signal at each user lies in the CI-regions of the respective transmitted symbols, for any PS error matrix $\mathbf{E} \in \mathcal{E}$. The corresponding problem can be formulated as a semi-infinite optimization problem [22, 33] given by

\footnotetext{
${ }^{1}$ We employ PSK-modulation here for simplicity. Nevertheless, the proposed techniques can be extended to other modulation formats following the principles in [28].
}

$$
\begin{aligned}
\underset{\mathbf{A},\left\{\mathbf{d}_{\ell}\right\}_{\ell \in \mathcal{K}}}{\operatorname{minimize}} & \left\|\mathbf{A} \sum_{\ell=1}^{K} \mathbf{d}_{k} s_{k}\right\|^{2} \\
\text { s.t. } & \left|\operatorname{Im}\left(s_{k}^{*} \tilde{\mathbf{h}}_{k}^{\top}(\mathbf{A}+\mathbf{E}) \sum_{\ell=1}^{K} \mathbf{d}_{\ell} s_{\ell}\right)\right| \leq \\
& \left(\operatorname{Re}\left(s_{k}^{*} \tilde{\mathbf{h}}_{k}^{\top}(\mathbf{A}+\mathbf{E}) \sum_{\ell=1}^{K} \mathbf{d}_{\ell} s_{\ell}\right)-\gamma_{k}\right) \tan \theta \\
\forall \mathbf{E} \in \mathcal{E}, \forall k \in \mathcal{K}, & \\
& \left|a_{n r}\right|=a, \quad \forall n \in \mathcal{N}, \forall r \in \mathcal{R},
\end{aligned}
$$

where $\theta \triangleq \pi / M$, and $\gamma_{k} \triangleq \Gamma_{k} / \sin \theta$, with $\Gamma_{k}$ indicating the threshold-margin ${ }^{2}$ at the $k$ th user. The objective function (3a) minimizes the total transmit power at the BS. The constraints in (3b) enforce the received signals to lie in the appropriate CIregions $^{3}$ at each user, $\forall \mathbf{E} \in \mathcal{E}$. The constraints in (3c) enforce the magnitude of each PS value in matrix $\mathbf{A}$ to its nominal value $a$. This problem is difficult to solve due to the following reasons: 1) bilinear coupling between the optimization variables in $\mathbf{A}$ and $\mathbf{d}_{k}, 2$ ) infinite number of constraints in (3b), 3) nonconvex constant magnitude constraint in (3c). To overcome these challenge, we decompose the problem into two stages, namely analog precoding and robust digital precoding, as in [22]. The analog precoding matrix is computed by employing any standard methods as discussed in $[21,22]$. Let $\hat{\mathbf{A}}$ denote the corresponding analog precoding matrix. By substituting $\mathbf{h}_{k} \triangleq s_{k}^{*} \tilde{\mathbf{h}}_{k}$, treating the composite precoding term $\sum_{\ell=1}^{K} \mathbf{d}_{\ell} s_{\ell}$ as a single precoder $\mathbf{b}$, and fixing the analog precoding matrix to $\hat{\mathbf{A}}$, problem (3) can be reformulated as

$$
\begin{aligned}
& \underset{\mathbf{b}}{\operatorname{minimize}}\|\hat{\mathbf{A}} \mathbf{b}\|^{2} \\
& \text { s. t. }\left|\operatorname{Im}\left(\mathbf{h}_{k}^{\top}(\hat{\mathbf{A}}+\mathbf{E}) \mathbf{b}\right)\right| \leq \\
& \left(\operatorname{Re}\left(\mathbf{h}_{k}^{\top}(\hat{\mathbf{A}}+\mathbf{E}) \mathbf{b}\right)-\gamma_{k}\right) \tan \theta, \forall \mathbf{E} \in \mathcal{E}, \forall k \in \mathcal{K} .
\end{aligned}
$$

Even though the above problem is convex, there are infinite linear constraints in (4b), which make the problem nontrivial. In the next section, we propose an iterative algorithm to solve the above problem efficiently.

Remark: There are two major differences between problem (4) and its counterpart in [22]. First, the error sets are different: In problem (4) the magnitude of the error element $e_{n r}$ is bounded by $\varrho$, i.e., $\left|e_{n r}\right| \leq \varrho$, while in [22] the error element $e_{n r}$ has unit magnitude and its phase is limited by $\delta$, i.e., $\left|e_{n r}\right|=1$ and $\left|\angle e_{n r}\right| \leq \delta$. Second, in problem (4) the error element $e_{n r}$ is additive to the corresponding analog precoder coefficient $a_{n r}$. In contrast, the error element $e_{n r}$ is multiplicative with $a_{n r}$ in [22].

\section{Proposed Algorithm}

We extend the iterative algorithm developed in [22]—which is based on the cutting plane method and alternating procedure

\footnotetext{
${ }^{2}$ The threshold-margin controls the quality of service (QoS) at the user. For more information, the readers are referred to [22].

${ }^{3}$ Due to the space constraints, the readers are referred to [17, 22] for the geometrical interpretation of this formulation.
} 


$$
\begin{aligned}
\underset{\mathbf{b}^{i}}{\operatorname{minimize}} & \left.\left\|\hat{\mathbf{A}} \mathbf{b}^{i}\right\|\right|^{2} \\
\text { s.t. } & +\operatorname{Im}\left(\mathbf{h}_{k}^{\top}(\hat{\mathbf{A}}+\mathbf{E}) \mathbf{b}^{i}\right) \leq\left(\operatorname{Re}\left(\mathbf{h}_{k}^{\top}(\hat{\mathbf{A}}+\mathbf{E}) \mathbf{b}^{i}\right)-\gamma_{k}\right) \tan \theta, \forall \mathbf{E} \in \mathcal{E}_{k}^{i+}, \forall k \in \mathcal{K}, \\
& -\operatorname{Im}\left(\mathbf{h}_{k}^{\top}(\hat{\mathbf{A}}+\mathbf{E}) \mathbf{b}^{i}\right) \leq\left(\operatorname{Re}\left(\mathbf{h}_{k}^{\top}(\hat{\mathbf{A}}+\mathbf{E}) \mathbf{b}^{i}\right)-\gamma_{k}\right) \tan \theta, \forall \mathbf{E} \in \mathcal{E}_{k}^{i-}, \forall k \in \mathcal{K} . \\
& \hat{f} \triangleq \operatorname{Im}\left(\mathbf{h}_{k}^{\top}(\hat{\mathbf{A}}+\mathbf{E}) \mathbf{b}^{i \star}\right)-\left(\operatorname{Re}\left(\mathbf{h}_{k}^{\top}(\hat{\mathbf{A}}+\mathbf{E}) \mathbf{b}^{i \star}\right)-\gamma_{k}\right) \tan \theta .
\end{aligned}
$$

$[34,35]$ - to efficiently solve the formulated semi-infinite problem (4) by exploiting a structure in the problem, namely, the bounded magnitude property of elements of error matrices $\forall \mathbf{E} \in \mathcal{E}$.

The proposed algorithm begins with initializing finite error matrix sets $\mathcal{E}_{k}^{i+}=\{\mathbf{0}\}$ and $\mathcal{E}_{k}^{i-}=\{\mathbf{0}\}, \forall k \in \mathcal{K}$ at iteration number $i=1$, where $\mathbf{0}$ is an $N \times R$ matrix with all elements being equal to 0 . The algorithm comprises the following two stages in each iteration.

Update Digital Precoder: In the first stage of the $i$ th iteration, we solve the convex quadratic problem (5), which corresponds to the non-robust precoding problem in the first iteration. In the subsequent iterations, this problem comprises a finite subset of constraints of problem (4): the constraint (5b) for every error matrix $\mathbf{E} \in \mathcal{E}_{k}^{i+}$; the constraint (5c) for every error matrix $\mathbf{E} \in$ $\mathcal{E}_{k}^{i-}, \forall k \in \mathcal{K}$. The problem (5) can be efficiently solved by employing the low-complexity parallel implementation scheme proposed in [22], or alternatively, using any general purpose solver such as CVX [36] and CPLEX [37]. Let $\mathbf{b}^{i \star}$ denote the optimal solution of problem (5) at the $i$ th iteration.

Update Worst-Case Error Matrix Sets: In the second stage of the $i$ th iteration, we compute the worst-case error matrices $\mathbf{E}_{k}^{i+}$ and $\mathbf{E}_{k}^{i-}$, for constraints in (4b) at $\mathbf{b}=\mathbf{b}^{i \star}$, which violate the constraints with the largest margins. We formulate the corresponding problems as convex programs given by

$$
\begin{aligned}
& \mathbf{E}_{k}^{i+}=\underset{\left|e_{n r}\right| \leq \varrho}{\operatorname{argmax}}(+\hat{f}), \\
& \mathbf{E}_{k}^{i-}=\underset{\left|e_{n r}\right| \leq \varrho}{\operatorname{argmax}}(-\hat{f}),
\end{aligned}
$$

where $\hat{f}$ is defined in Eq. (6). In the following, we derive closed-form expressions for $\mathbf{E}_{k}^{i+}$ and $\mathbf{E}_{k}^{i-}$ for efficiently computing these matrices.

Consider the objective function $\hat{f}$ of problem (7). Let $g \triangleq$ $\mathbf{h}_{k}^{\top}(\hat{\mathbf{A}}+\mathbf{E}) \mathbf{b}^{i \star}$. We can rewrite $g$ as

$$
g=\underbrace{\mathbf{h}_{k}^{\top} \hat{\mathbf{A}} \mathbf{b}^{i \star}}_{\text {constant }}+\sum_{\forall n \in \mathcal{N}} \sum_{\forall r \in \mathcal{R}} h_{n k} b_{r}^{i \star} e_{n r} .
$$

The above expression for $g$ reveals that the objective function $\hat{f}$ is separable in each optimization variable $e_{n r}$. Therefore, the function $\hat{f}$ can be maximized independently and separately with respect to (w.r.t.) each $e_{n r}$ for $n \in \mathcal{N}, r \in \mathcal{R}$. Consider a summand $h_{n k} b_{r}^{i \star} e_{n r}$ of $g$. Define $\bar{\chi}+\mathrm{j} \tilde{\chi} \triangleq h_{n k} b_{r}^{i \star}$ and $\alpha+\mathrm{j} \beta \triangleq e_{n r}$, where $\mathrm{j}$ denotes the imaginary unit. Substituting these new definitions, the term of function $\hat{f}$ that comprises the variable $e_{n r}$ can be expressed as

$$
\tilde{f}(\alpha, \beta)=\underbrace{(\tilde{\chi}-\bar{\chi} \tan \theta)}_{\kappa} \alpha+\underbrace{(\bar{\chi}+\tilde{\chi} \tan \theta)}_{\tau} \beta .
$$

The constraints on the magnitude of the PS errors in problem (7), given by $\left|e_{n r}\right| \leq \varrho$, can be equivalently expressed in terms of $\alpha$ and $\beta$ as $\alpha^{2}+\beta^{2} \leq \varrho^{2}$. Accordingly, the optimization problem for maximizing function $\hat{f}$ w.r.t. $e_{n r}$ can be formulated as

$$
\begin{aligned}
\underset{\alpha, \beta}{\operatorname{maximize}} & \kappa \alpha+\tau \beta \\
\text { s. } & \text { t. } \alpha^{2}+\beta^{2} \leq \varrho^{2} .
\end{aligned}
$$

Note that, for a finite $\kappa, \tau$, and $\varrho$, the above problem is always feasible and bounded above. However, if the constraint (11b) is removed, then the above problem becomes unbounded. Therefore, we can argue that the constraint (11b) is an active constraint and at optimum, the constraint is satisfied with equality, ${ }^{4}$ i.e., $\alpha^{2}+\beta^{2}=\varrho^{2}$. Now, substituting $\beta= \pm \sqrt{\varrho^{2}-\alpha^{2}}$ in Eq. (10), we obtain the function

$$
f(\alpha)=\kappa \alpha \pm \tau \sqrt{\varrho^{2}-\alpha^{2}} .
$$

This function comprises the following two variants:

$$
\begin{aligned}
& f_{1}(\alpha)=\kappa \alpha+\tau \sqrt{\varrho^{2}-\alpha^{2}}, \\
& f_{2}(\alpha)=\kappa \alpha-\tau \sqrt{\varrho^{2}-\alpha^{2}} .
\end{aligned}
$$

Differentiating Eq. (13a) and (13b) w.r.t. $\alpha$ and equating them to zero, we obtain the maximizer $\alpha^{\star}$ of the functions $f_{1}$ and $f_{2}$, as

$$
\alpha^{\star}=\frac{\kappa \varrho}{\sqrt{\kappa^{2}+\tau^{2}}}=\frac{\varrho(\tilde{\chi} \cos \theta-\bar{\chi} \sin \theta)}{|\bar{\chi}+\mathrm{j} \tilde{\chi}|} .
$$

Consequently, $\beta^{\star}$ can be expressed as

$$
\beta^{\star}=\operatorname{sign}(\tau) \sqrt{\varrho^{2}-\alpha^{\star 2}}=\frac{\bar{\chi}+\tilde{\chi} \tan \theta}{|\bar{\chi}+\tilde{\chi} \tan \theta|} \sqrt{\varrho^{2}-\alpha^{\star 2}} .
$$

Now we define $\mathbf{Z} \triangleq \mathbf{h}_{k}\left(\mathbf{b}^{i \star}\right)^{\top}$. Then, the worst-case error values of all PSs can be obtained efficiently by computing the error matrix $\mathbf{E}_{k}^{+}=\mathbf{U}^{+}+\mathrm{j} \mathbf{W}^{+}$, where

$$
\begin{aligned}
& u_{n r}^{+}=\frac{\varrho\left(\operatorname{Im}\left(z_{n r}\right) \cos \theta-\operatorname{Re}\left(z_{n r}\right) \sin \theta\right)}{\left|z_{n r}\right|}, \\
& w_{n r}^{+}=\frac{\operatorname{Re}\left(z_{n r}\right)+\operatorname{Im}\left(z_{n r}\right) \tan \theta}{\left|\operatorname{Re}\left(z_{n r}\right)+\operatorname{Im}\left(z_{n r}\right) \tan \theta\right|} \sqrt{\varrho^{2}-\left(u_{n r}^{+}\right)^{2}} .
\end{aligned}
$$

Similarly, we can derive a closed-form expression for the optimal solution of problem (8) as $\mathbf{E}_{k}^{-}=\mathbf{U}^{-}+\mathbf{j} \mathbf{W}^{-}$, where

$$
\begin{aligned}
& u_{n r}^{-}=\frac{\varrho\left(-\operatorname{Im}\left(z_{n r}\right) \cos \theta-\operatorname{Re}\left(z_{n r}\right) \sin \theta\right)}{\left|z_{n r}\right|}, \\
& w_{n r}^{-}=\frac{-\operatorname{Re}\left(z_{n r}\right)+\operatorname{Im}\left(z_{n r}\right) \tan \theta}{\left|-\operatorname{Re}\left(z_{n r}\right)+\operatorname{Im}\left(z_{n r}\right) \tan \theta\right|} \sqrt{\varrho^{2}-\left(u_{n r}^{-}\right)^{2}} .
\end{aligned}
$$

${ }^{4}$ For $M=2, \tan \theta=\infty$. In this case, the constraint (4b) and problem (11) can be reformulated to omit $\tan \theta$, and subsequently arrive at the same conclusion that the constraint (11b) is satisfied with the equality. 


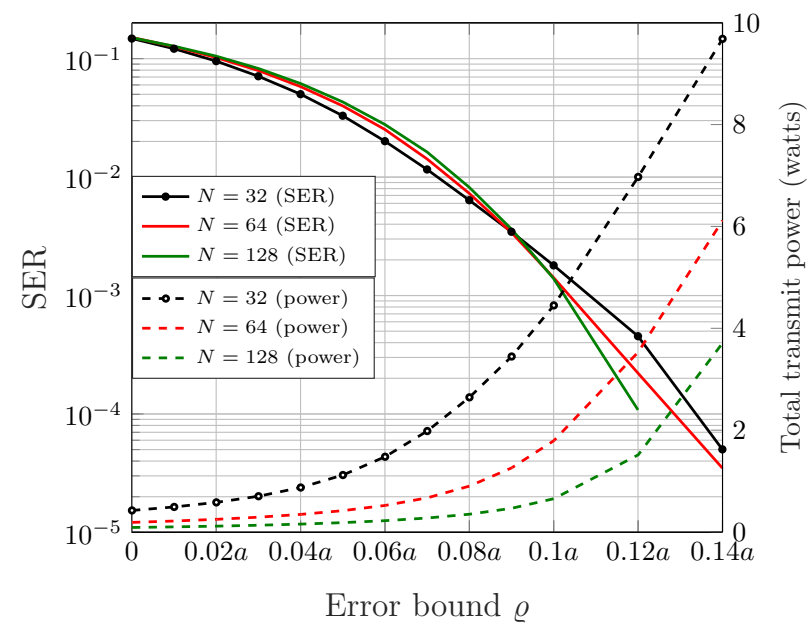

Fig. 3: SER and total transmit power vs. error bound $\varrho$ for $R=K=M=4, \mathrm{TNR}=1$, and $\mathrm{CPC}$ analog precoding.

Now, if $\mathbf{E}_{k}^{i+}$ violates the constraint (4b), then it is added to the error matrix $\mathcal{E}_{k}^{i+}$, i.e., $\mathcal{E}_{k}^{(i+1)+}=\mathcal{E}_{k}^{i+} \cup \mathbf{E}_{k}^{i+}$. Similarly, if the error matrix $\mathbf{E}_{k}^{i-}$ violates the constraint (4b), then it is included in set $\mathcal{E}_{k}^{(i+1)-}$, i.e., $\mathcal{E}_{k}^{(i+1)-}=\mathcal{E}_{k}^{i-} \cup \mathbf{E}_{k}^{i-}$. When both $\mathbf{E}_{k}^{i+}$ and $\mathbf{E}_{k}^{i-}, \forall k \in \mathcal{K}$, satisfy the constraints (4b), the vector $\mathbf{b}^{i \star}$ fulfills all constraints in (4b), and hence the algorithm is terminated.

\section{NuMERICAL RESULTS}

For the simulation, we employed a BS equipped with $R=4$ RF chains, QPSK modulation, and $K=4$ users. We used the geometric channel model with 15 propagation paths [22]. The nominal magnitude of each PS is given by $a=\frac{1}{\sqrt{N}}$. The PS errors $e_{n r}, \forall n \in \mathcal{N}, \forall r \in \mathcal{R}$ are distributed uniformly within a disk with center at the origin and radius of $\varrho$.

Table I lists the average SER increase due to the phase and magnitude errors when the non-robust CI-based hybrid precoding scheme in [21] is employed for different values of threshold-margin-to-noise power ratio (TNR). In the table, we note that increase of the error bound $\varrho$ corresponds to a drastic increase in the SER. ${ }^{5}$ In particular, for larger values of TNR, the increase in SER is unacceptably high for critical applications (up to approx. $14 \%$ for $\varrho=0.3 a$ and TNR $=2.5$ ).

TABLE I: SER increase due to PS errors in the case of nonrobust CI-based hybrid precoding for $N=128, R=K=$ $M=4$, and CPC analog precoding [21].

\begin{tabular}{|c||c|c|c|c|}
\hline$\varrho$ & TNR = 1.0 & TNR = 1.5 & TNR = 2.0 & TNR = 2.5 \\
\hline \hline $0.1 a$ & $0.08 \%$ & $0.36 \%$ & $1.02 \%$ & $0.42 \%$ \\
\hline $0.2 a$ & $0.24 \%$ & $0.97 \%$ & $3.51 \%$ & $4.24 \%$ \\
\hline $0.3 a$ & $0.55 \%$ & $2.45 \%$ & $7.48 \%$ & $13.94 \%$ \\
\hline
\end{tabular}

Figure 3 plots the achieved SER and the corresponding transmit power (in watts) over a range of error bound values $\varrho$ for different numbers of transmit antennas $N$. In the figure, we observe that the transmit power associated with the robust

\footnotetext{
${ }^{5}$ In the results, the error bounds are specified in terms of nominal magnitude of the PSs $a$.
}

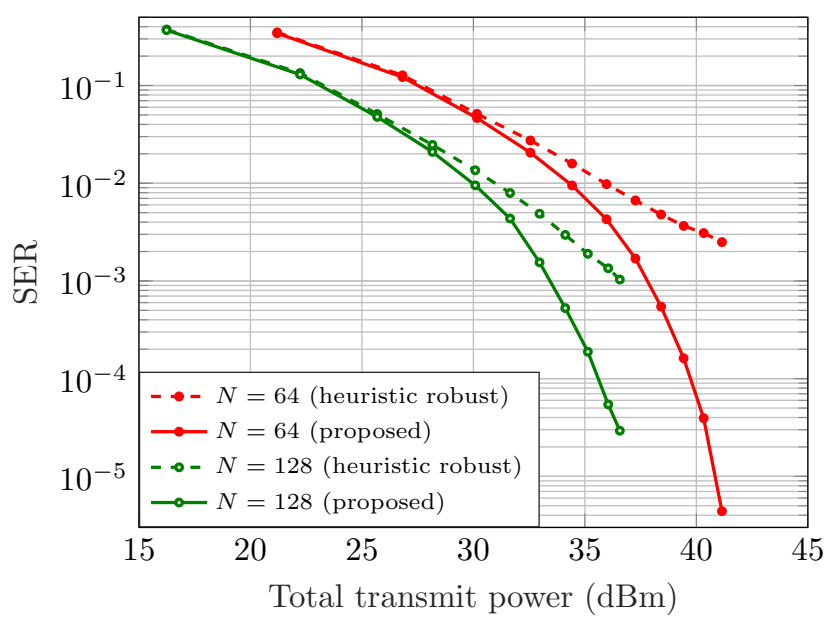

Fig. 4: Performance comparison of the robust precoding techniques for $R=K=M=4$, and CPC analog precoding.

precoders increases, whereas the resulting SER decreases, as the error bound $\varrho$ increases. The robust precoders with larger transmit powers - which are primarily designed to circumvent the undesired effect of PS errors-also reduce the errors caused by the additive noise at the users. In the figure, we also notice that as the number of transmit antennas increases, the required transmit power significantly reduces to achieve a given QoS.

Figure 4 compares the performance of the proposed robust hybrid precoding scheme with that of a conventional heuristic robust precoding technique, where the transmit powers of the non-robust precoders are linearly scaled to meet the required QoS [38, 39]. In the simulation, we compute the required transmit power and the resulting SER by the proposed robust hybrid precoding scheme for a range of TNR values. Afterward, the non-robust CI-based hybrid precoders $(\varrho=0)$ are computed, and their powers are scaled to match them to the transmit powers achieved by the proposed scheme. Finally, the corresponding SERs are computed. The simulations are carried out for the scenario where the PSs suffer the worst-case errors for at-least one user. The figure demonstrates that for a given transmit power budget the proposed robust precoding scheme achieves a better SER performance when compared to the conventional robust precoding technique.

\section{CONCLUSION}

In this paper, we considered the hybrid analog-digital precoding in a multiuser massive MIMO system, where the PSs in the hybrid precoding architecture are imperfect. We formulated a semi-infinite problem to compute the robust digital precoders that are robust against both phase and magnitude errors associated with PSs. Subsequently, we presented an iterative algorithm, where the precoders and the worstcase error matrices are updated sequentially and iteratively. We derived the closed-form expressions for the worst-case error matrices by exploiting the special structure in the error model. The numerical results demonstrated that the proposed algorithm significantly reduces the SER when compared to the non-robust hybrid precoding. 


\section{REFERENCES}

[1] T. L. Marzetta, "Massive MIMO: An introduction," Bell Labs Technical Journal, vol. 20, pp. 11-22, Mar. 2015.

[2] F. Rusek et al., "Scaling up MIMO: Opportunities and challenges with very large arrays," IEEE Signal Process. Mag., vol. 30, no. 1, pp. 40-60, Jan. 2013.

[3] J. Hoydis, S. ten Brink, and M. Debbah, "Massive MIMO in the UL/DL of cellular networks: How many antennas do we need?" IEEE J. Select. Areas Commun., vol. 31, no. 2, pp. 160-171, Feb. 2013.

[4] L. Lu et al., "An overview of massive MIMO: Benefits and challenges," IEEE J. Select. Topics in Signal Process., vol. 8, no. 5, pp. 742-758, Oct. 2014.

[5] J. Mietzner et al., "Multiple-antenna techniques for wireless communications-A comprehensive literature survey," IEEE Commun. Surveys Tutorials, vol. 11, no. 2, pp. 87-105, Feb. 2009.

[6] M. Bengtsson and B. Ottersten, "Optimal and suboptimal transmit beamforming," Handbook of Antennas in Wireless Commun., CRC Press, pp. 18-1-18-33, Aug. 2001, doi:10.1201/9781420042146.

[7] M. Schubert and H. Boche, "Solution of the multiuser downlink beamforming problem with individual SINR constraints," IEEE Trans. Veh. Technol., vol. 53, no. 1, pp. 18-28, Jan. 2004.

[8] A. F. Molisch et al., "Hybrid beamforming for massive MIMO: A survey," IEEE Commun. Mag., vol. 55, no. 9, pp. 134-141, Sep. 2017.

[9] M. Shafi et al., "5G: A tutorial overview of standards, trials, challenges, deployment, and practice," IEEE J. Select. Areas Commun., vol. 35, no. 6, pp. 1201-1221, Jun. 2017.

[10] L. Liang, W. Xu, and X. Dong, "Low-complexity hybrid precoding in massive multiuser MIMO systems," IEEE Wireless Commun. Letters, vol. 3, no. 6, pp. 653-656, Dec. 2014.

[11] F. Sohrabi and W. Yu, "Hybrid digital and analog beamforming design for large-scale antenna arrays," IEEE J. Select. Topics in Signal Process., vol. 10, no. 3, pp. 501-513, Apr. 2016.

[12] X. Zhang, A. F. Molisch, and S. Y. Kung, "Variable-phase-shiftbased RF-baseband codesign for MIMO antenna selection," IEEE Trans. Signal Process., vol. 53, no. 11, pp. 4091-4103, Nov. 2005.

[13] G. Hegde and M. Pesavento, "Joint user selection and hybrid analog-digital beamforming in massive MIMO systems," in Proc. IEEE Sensor Array and Multichannel Signal Process. Workshop (SAM), Sheffield, UK, Jul. 2018.

[14] T. E. Bogale and L. B. Le, "Beamforming for multiuser massive MIMO systems: Digital versus hybrid analog-digital," in Proc. IEEE Global Commun. Conf. (GLOBECOM), Austin, TX, USA, Dec. 2014, pp. 4066-4071.

[15] G. Hegde, Y. Cheng, and M. Pesavento, "Hybrid beamforming for large-scale MIMO systems using uplink-downlink duality," in Proc. IEEE Int. Conf. on Acoustics, Speech and Signal Process. (ICASSP), New Orleans, USA, Mar. 2017.

[16] F. Sohrabi and W. Yu, "Hybrid analog and digital beamforming for mmWave OFDM large-scale antenna arrays," IEEE J. Select. Areas Commun., vol. 35, no. 7, pp. 1432-1443, Jul. 2017.

[17] C. Masouros and G. Zheng, "Exploiting known interference as green signal power for downlink beamforming optimization," IEEE Trans. Signal Process., vol. 63, no. 14, pp. 3628-3640, Jul. 2015.

[18] C. Masouros et al., "Known interference in the cellular downlink: A performance limiting factor or a source of green signal power?" IEEE Commun. Mag., vol. 51, no. 10, pp. 162-171, Oct. 2013

[19] G. Zheng et al., "Rethinking the role of interference in wireless networks," IEEE Commun. Mag., vol. 52, no. 11, pp. 152-158, Nov. 2014.

[20] S. Timotheou, G. Zheng, C. Masouros, and I. Krikidis, "Exploiting constructive interference for simultaneous wireless information and power transfer in multiuser downlink systems,"
IEEE J. Select. Areas Commun., vol. 34, no. 5, pp. 1772-1784, May 2016.

[21] G. Hegde, C. Masouros, and M. Pesavento, "Analog beamformer design for interference exploitation based hybrid beamforming," in Proc. IEEE Sensor Array and Multichannel Signal Process. Workshop (SAM), Sheffield, UK, Jul. 2018.

[22] G. Hegde, C. Masouros, and M. Pesavento, "Interference exploitation-based hybrid precoding with robustness against phase errors," IEEE Trans. Wireless Commun., vol. 18, no. 7, pp. 3683-3696, Jul. 2019.

[23] G. Hegde, C. Masouros, and M. Pesavento, "Coordinated hybrid precoding for interference exploitation in heterogeneous networks," IEEE Commun. Letters, pp. 1-1, 2019, doi:10.1109/LCOMM.2019.2933840.

[24] P. V. Amadori and C. Masouros, "Large scale antenna selection and precoding for interference exploitation," IEEE Trans. Commun., vol. 65, no. 10, pp. 4529-4542, Oct. 2017.

[25] K. Koh and G. M. Rebeiz, "0.13- $\mu$ m CMOS phase shifters for $\mathrm{X}-$, Ku-, and K-band phased arrays," IEEE Journal of SolidState Circuits, vol. 42, no. 11, pp. 2535-2546, Nov. 2007.

[26] W. T. Li et al., "60-GHz 5-bit phase shifter with integrated VGA phase-error compensation," IEEE Trans. on Microwave Theory and Techniques, vol. 61, no. 3, pp. 1224-1235, Mar. 2013.

[27] D. W. Kang, H. D. Lee, C. H. Kim, and S. Hong, "Ku-band MMIC phase shifter using a parallel resonator with $0.18-\mu \mathrm{m}$ CMOS technology," IEEE Trans. on Microwave Theory and Techniques, vol. 54, no. 1, pp. 294-301, Jan. 2006.

[28] A. Li and C. Masouros, "Exploiting constructive mutual coupling in P2P MIMO by analog-digital phase alignment," IEEE Trans. Wireless Commun., vol. 16, no. 3, pp. 1948-1962, Mar. 2017.

[29] M. A. Morton et al., "Sources of phase error and design considerations for silicon-based monolithic high-pass/low-pass microwave phase shifters," IEEE Trans. on Microwave Theory and Techniques, vol. 54, no. 12, pp. 4032-4040, Dec. 2006.

[30] W. Li et al., "60-GHz 5-bit phase shifter with integrated VGA phase-error compensation," IEEE Trans. on Microwave Theory and Techniques, vol. 61, no. 3, Mar. 2013.

[31] Y. Yu et al., "A $60 \mathrm{GHz}$ phase shifter integrated with LNA and PA in $65 \mathrm{~nm}$ CMOS for phased array systems," IEEE Journal of Solid-State Circuits, vol. 45, no. 9, pp. 1697-1709, Sep. 2010.

[32] H. Yin, D. Gesbert, M. Filippou, and Y. Liu, "A coordinated approach to channel estimation in large-scale multiple-antenna systems," IEEE J. Select. Areas Commun., vol. 31, no. 2, pp. 264-273, Feb. 2013.

[33] O. Stein, "How to solve a semi-infinite optimization problem," European Journal of Operational Research, vol. 223, no. 2, pp. 312-320, Dec. 2012.

[34] S. A. Gustafson and K. O. Kortanek, "Numerical treatment of a class of semi-infinite programming problems," Naval Research Logistics Quarterly, vol. 20, no. 3, pp. 477-504, 1973.

[35] S. Y. Wu, S. C. Fang, and C. J. Lin, "Relaxed cutting plane method for solving linear semi-infinite programming problems," Journal of Optimization Theory and Applications, vol. 99, no. 3, pp. 759-779, 1998.

[36] M. Grant and S. Boyd, "CVX: Matlab software for disciplined convex programming, version 2.1," http://cvxr.com/cvx, Mar. 2014.

[37] IBM, IBM ILOG CPLEX Optimization Studio CPLEX user's manual, 2011.

[38] J. Wang and D. P. Palomar, "Worst-case robust MIMO transmission with imperfect channel knowledge," IEEE Trans. Signal Process., vol. 57, no. 8, pp. 3086-3100, Aug. 2009.

[39] A. Pascual-Iserte, D. P. Palomar, A. I. Perez-Neira, and M. A. Lagunas, "A robust maximin approach for MIMO communications with imperfect channel state information based on convex optimization," IEEE Trans. Signal Process., vol. 54, no. 1, pp. 346-360, Jan. 2006. 